\title{
ATLAS measurements of vector boson production, inclusive and with associated jets
}

QCD 14

Montpellier (France), 30 June - 4 July, 2014

Nicola Orlando on behalf of the ATLAS Collaboration

INFN Lecce,

Dipartimento di Matematica e Fisica "Ennio De Giorgi", Universita' del Salento University College London
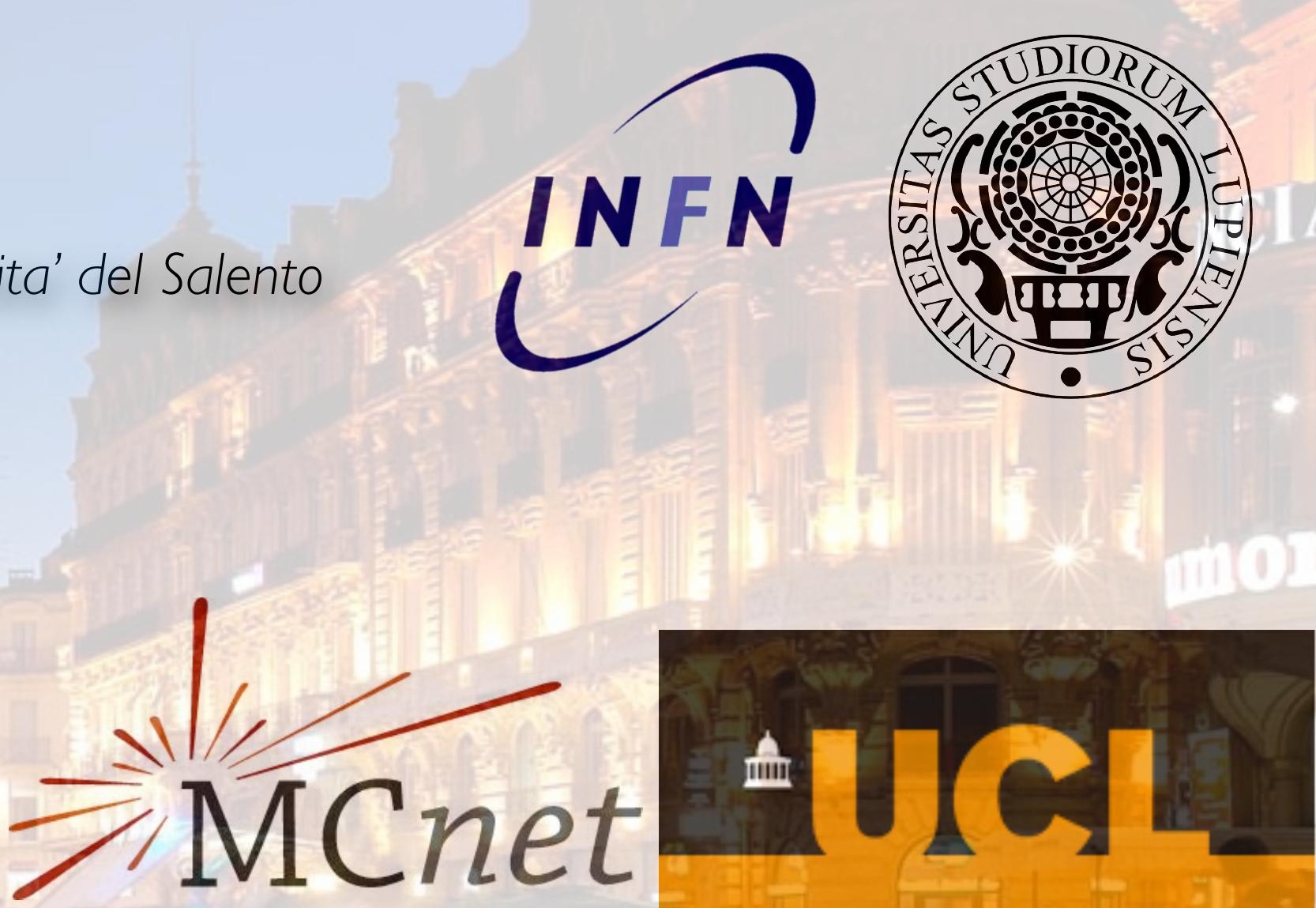


\section{Outline}

Inclusive gauge boson production

■ Z transverse momentum (arXiv: I 406.3660)

曰 Low mass Drell-Yan (arXiv: I 404.I 2 I 2)

$\square$ Gauge boson production in association with jets

G Z+jets (JHEP07(20 I 3)032)

- Gauge boson production in association with heavy flavors

■ W+charm (JHEP | 405 (20|4) 068)

G W+b (JHEP 06 (20 I 3) 084)

घ Z+b-jets (Preliminary)

口 Summary 


\section{Inclusive gauge boson production}

Рт(II) at the $Z$ peak

口 Experimental final state with high purity

口 Z transverse momentum measurement to access to QCD radiation in all kinematics regimes

口 Di-lepton pair production at the $Z$ pole is known to constrain the quark parton density functions

घ Explore out of peak regions to probe different different ranges of $\left(x, Q^{2}\right)$ and quark mixtures

Available theoretical tools provide accuracy up to NNLO QCD and can include EW corrections up to NLO or resummation of $Q C D$ radiation up to NNLL

घ MC based on LO matrix element +PS, NLO+PS also used

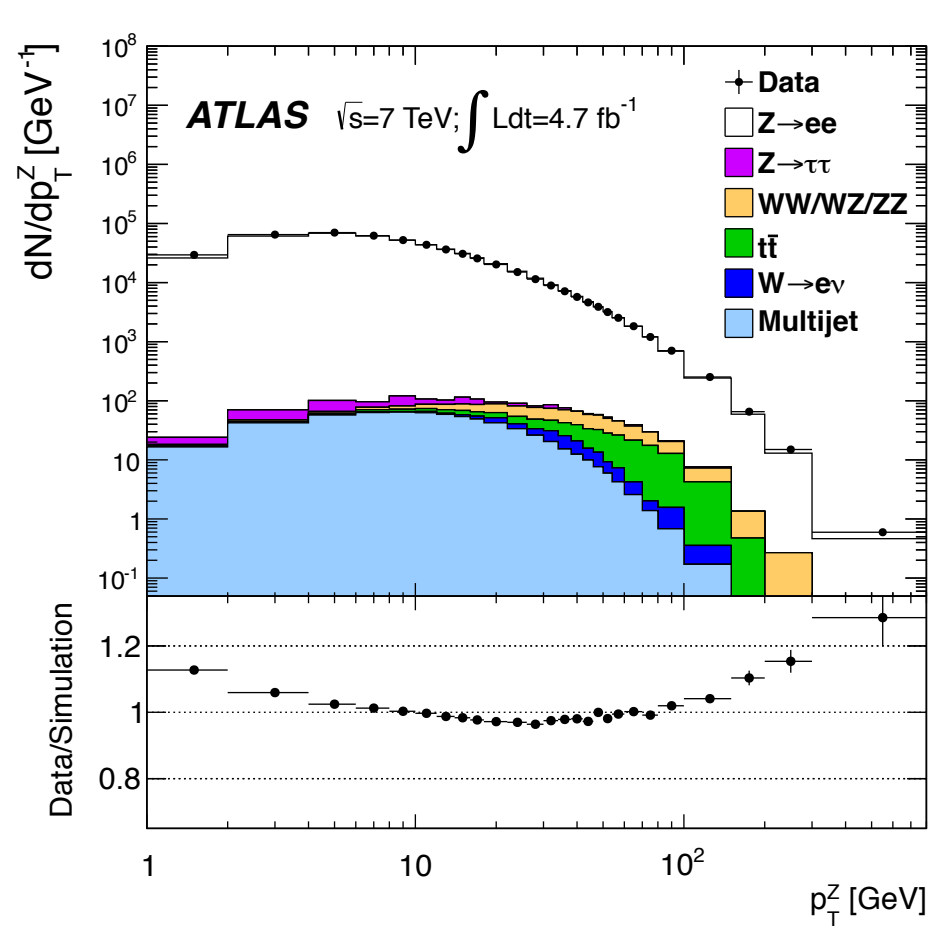

Drell-Yan at low mass

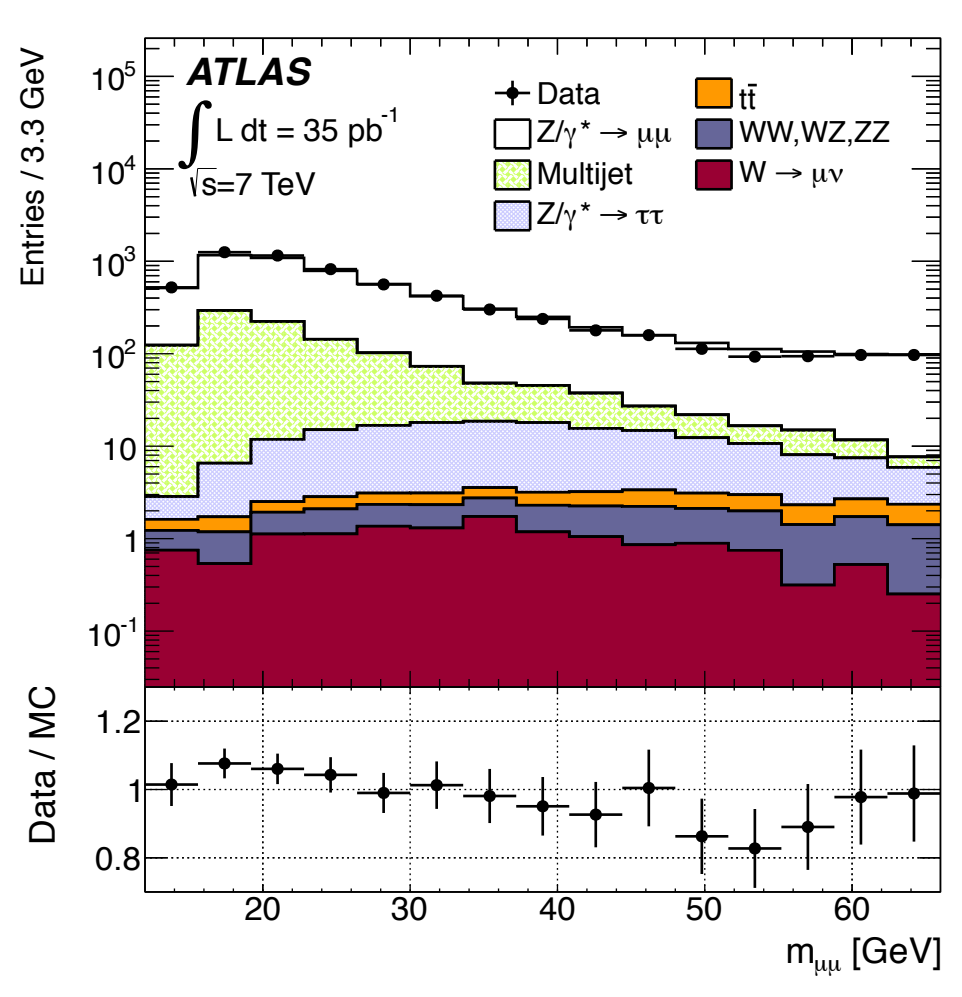




\section{$\mathbf{Z}$ boson transverse momentum}

$\square \quad$ Fixed order NNLO QCD underestimate the measured cross section by about $10 \%$ in the intermediate $\mathrm{PT}(\mathrm{Z})$ region

口 Transverse momentum shape reasonably well described above $\sim 30 \mathrm{GeV}$

NLO EW corrections affect the high PT(Z) tail only

․ Experimental precision of about $\sim 0.5 \%$
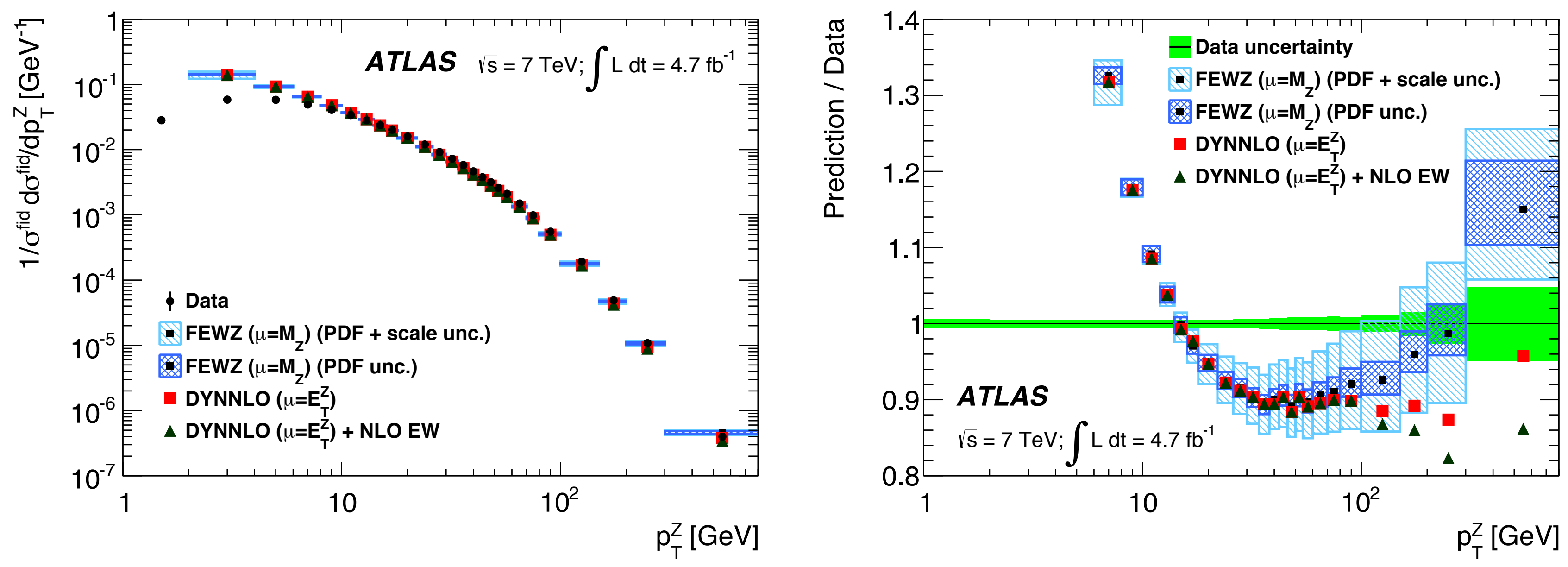


\section{$\mathbf{Z}$ boson transverse momentum}

$\square$ Data compared to other predictions, including NLL or NNLL resummation

घ Low Pт(Z) region well described

$\square$ Residual data-theory differences almost covered by theory errors
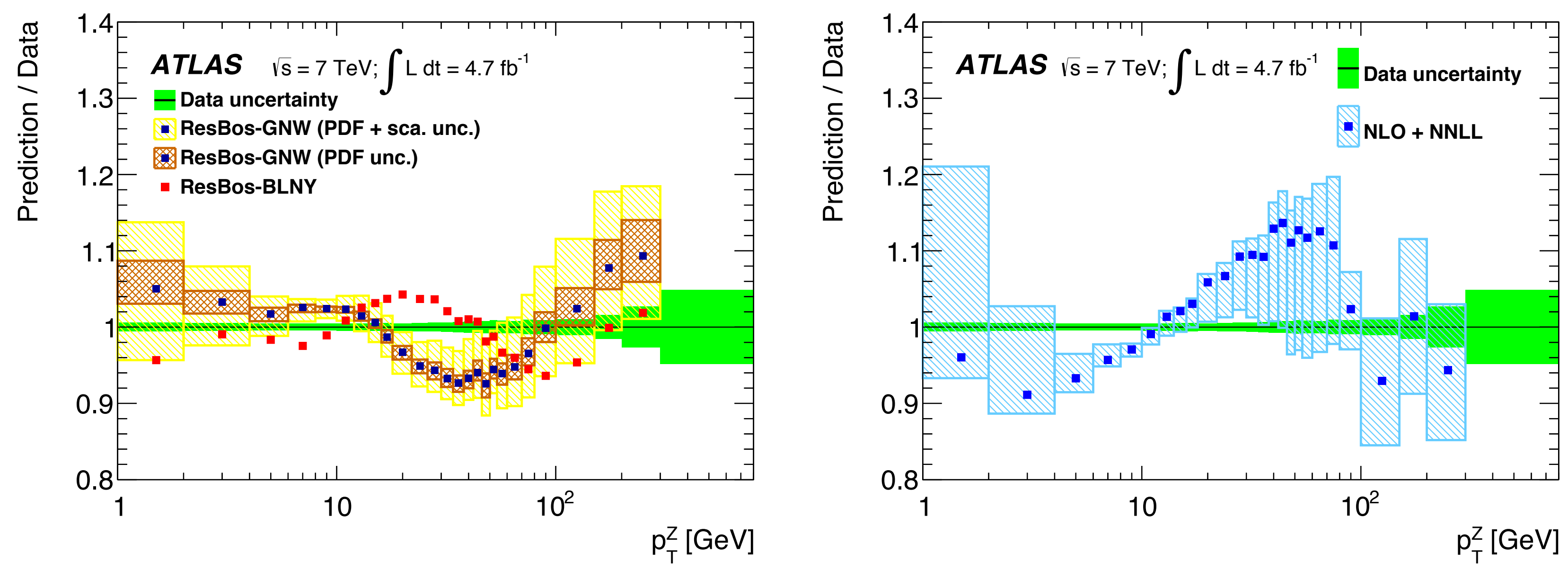


\section{Low mass neutral current Drell-Yan}

- Access to di-lepton invariant mass down to $12 \mathrm{GeV}$

口 Fixed order NNLO QCD + NLO EW + PI (photon induced dilepton pair production) describes well the data
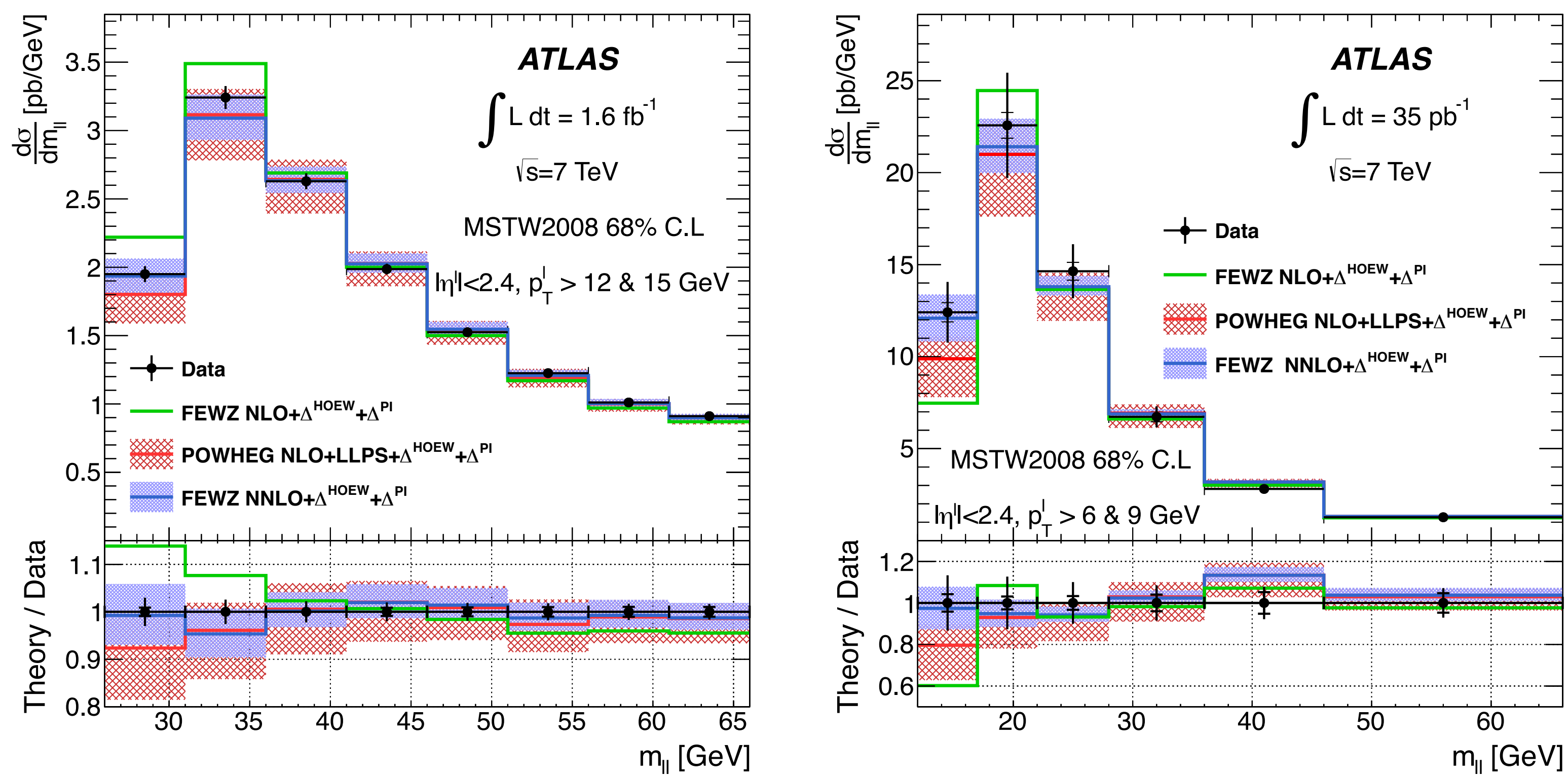

Nicola Orlando - QCD 14, Montpellier, 30 June - 4 July, 2014 


\section{Gauge bosons production in association with jets}

- Probe of high PT QCD radiation description, background for Higgs measurements and New Physics searches

ㄱ Z+jets cross section: good statistics ( $\sim 10^{5}$ candidate events), small background

- Measurements also motivated by continuous theoretical progresses

$\square$ State-of-the-art predictions include LO multileg+PS, NLO,

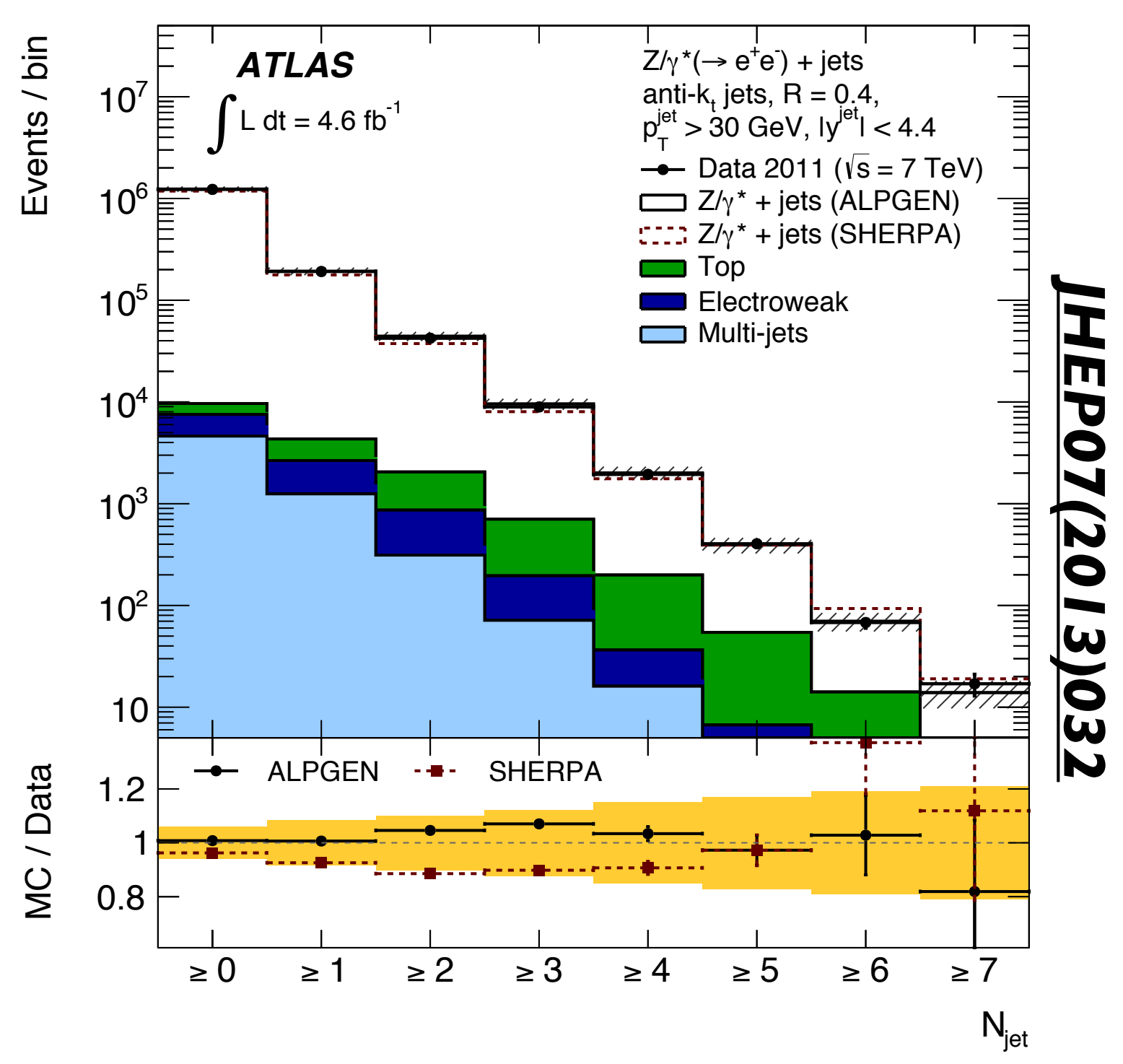
$\mathrm{NLO}+\mathrm{PS}$, fixed order NLO (for $\mathrm{V}+\mathrm{Njets)}$ 


\section{Z+jets}

口 In a nutshell: the data are well described by theory predictions using multileg $M C$ or NLO multileg calculations

घ Some expected limitations or some small "features" of the currently used MC
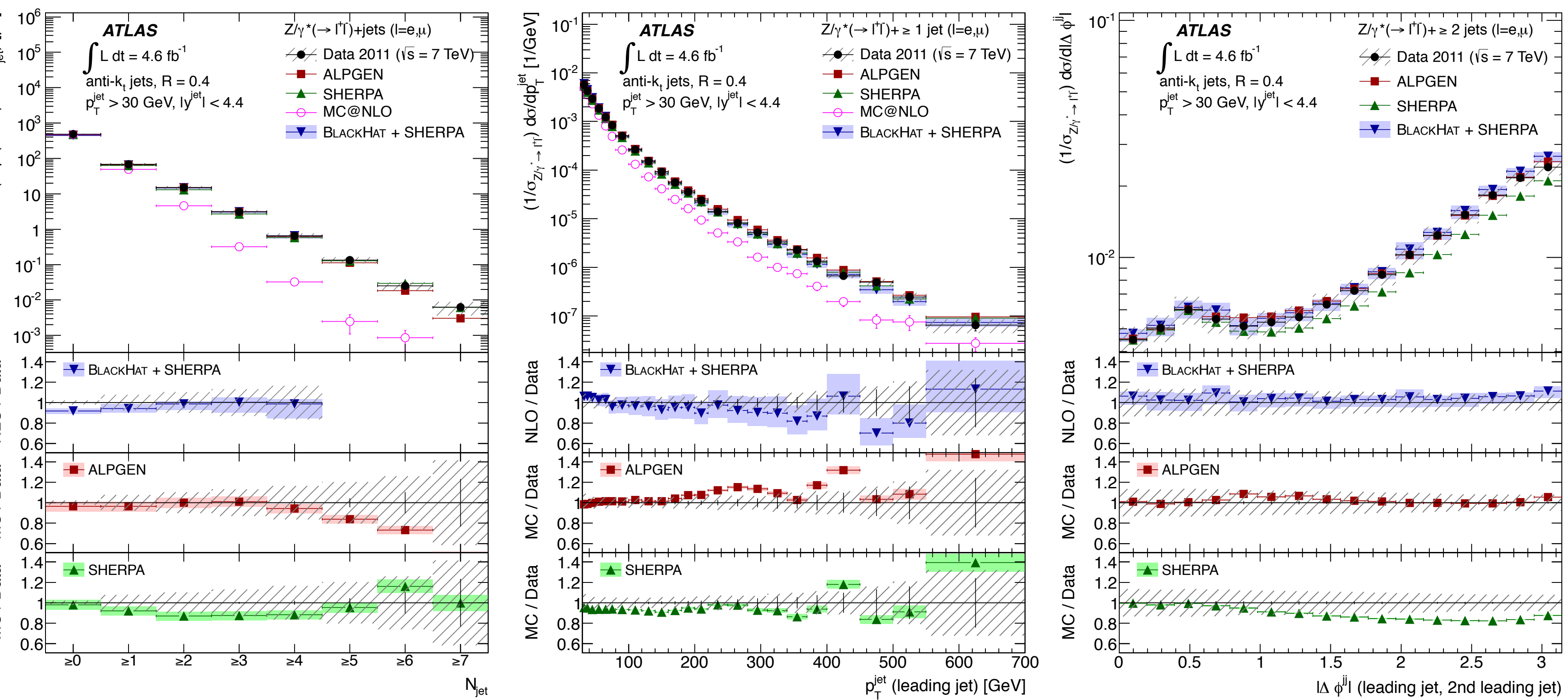

Nicola Orlando - QCD 14, Montpellier, 30 June - 4 July, 2014 


\section{Gauge bosons production in association with heavy flavors}

$\square \quad$ The associated production of gauge bosons and heavy flavors has a rich phenomenology at LHC

घ Sensitive to parton distribution functions

$\square \quad$ Interesting for testing various aspects of $M C$ modeling

$\square \quad$ Exploiting dedicated analysis strategies to face the large backgrounds

․ Available theoretical predictions: NLO+PS, LO multileg +PS, fixed order NLO
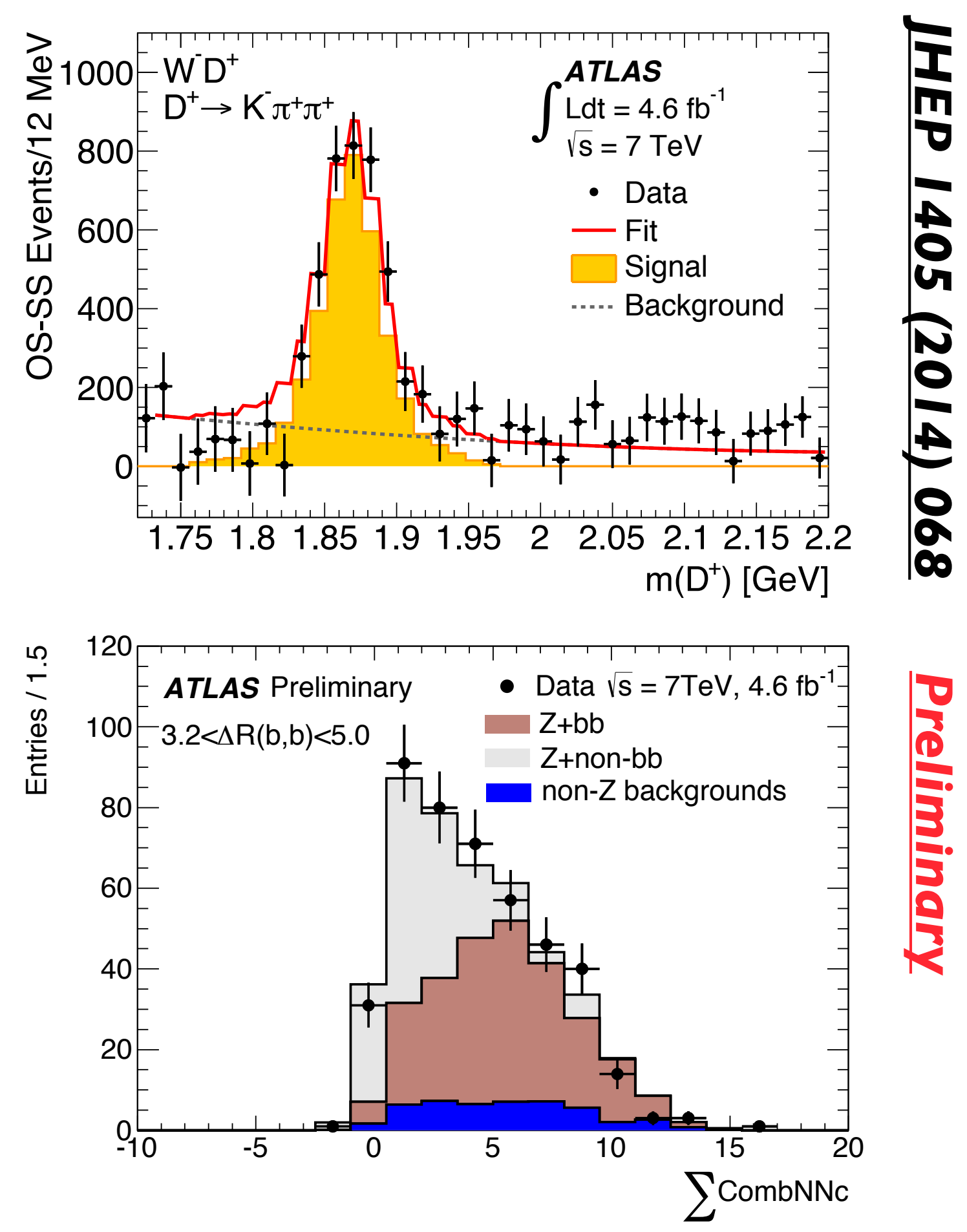


\section{W+charm: total fiducial cross sections}

口 Comparing W+charm-jets and W+D measurements to NLO+PS interfaced to several PDF sets

छ Testing suppressed vs unsuppressed scenarios for the strange quark PDF

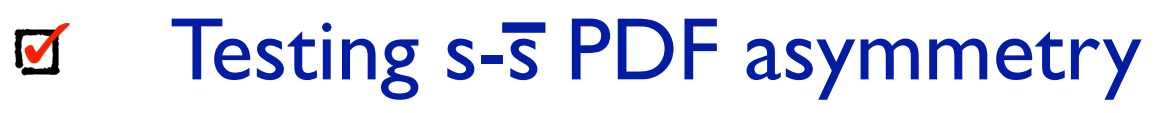

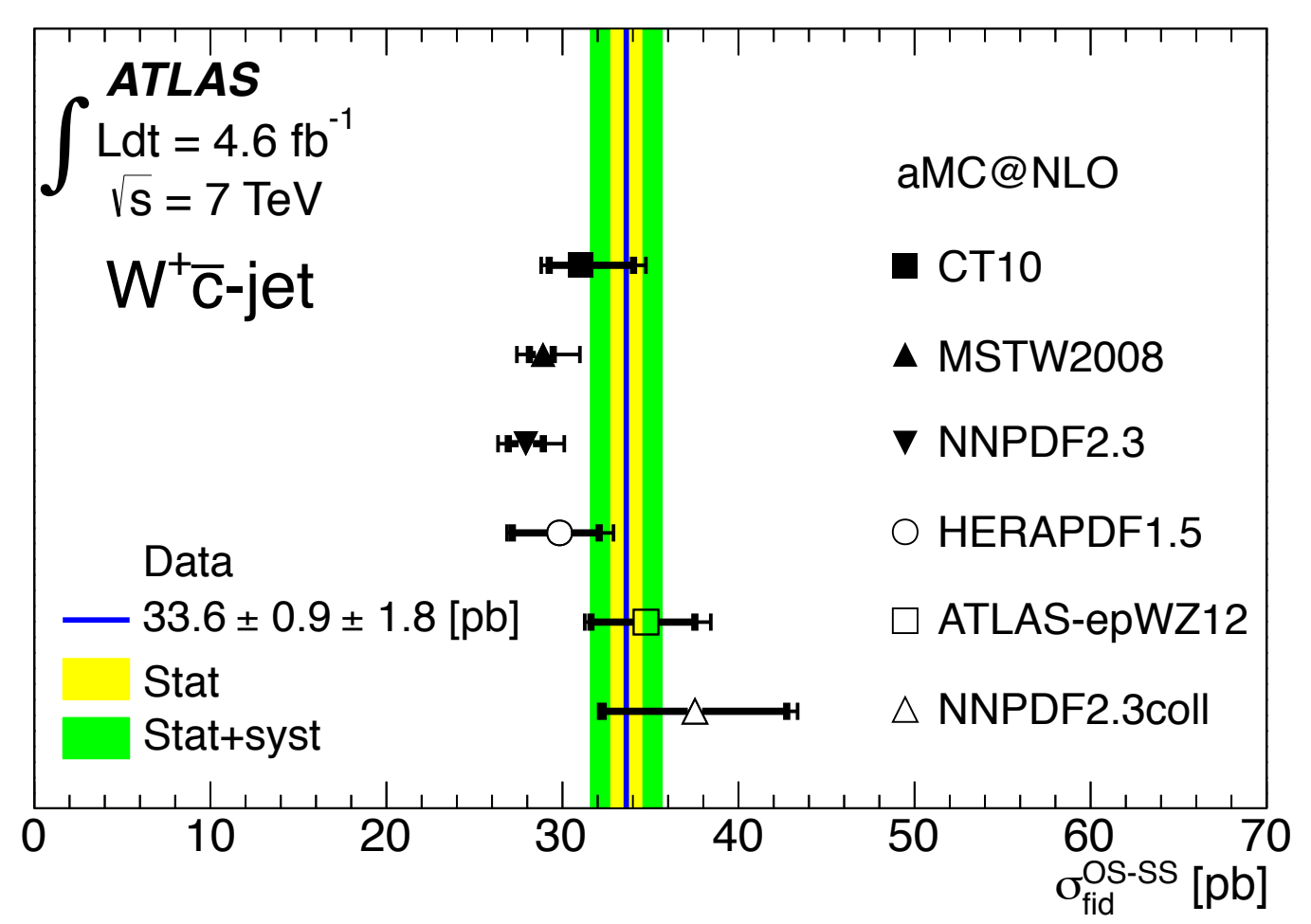

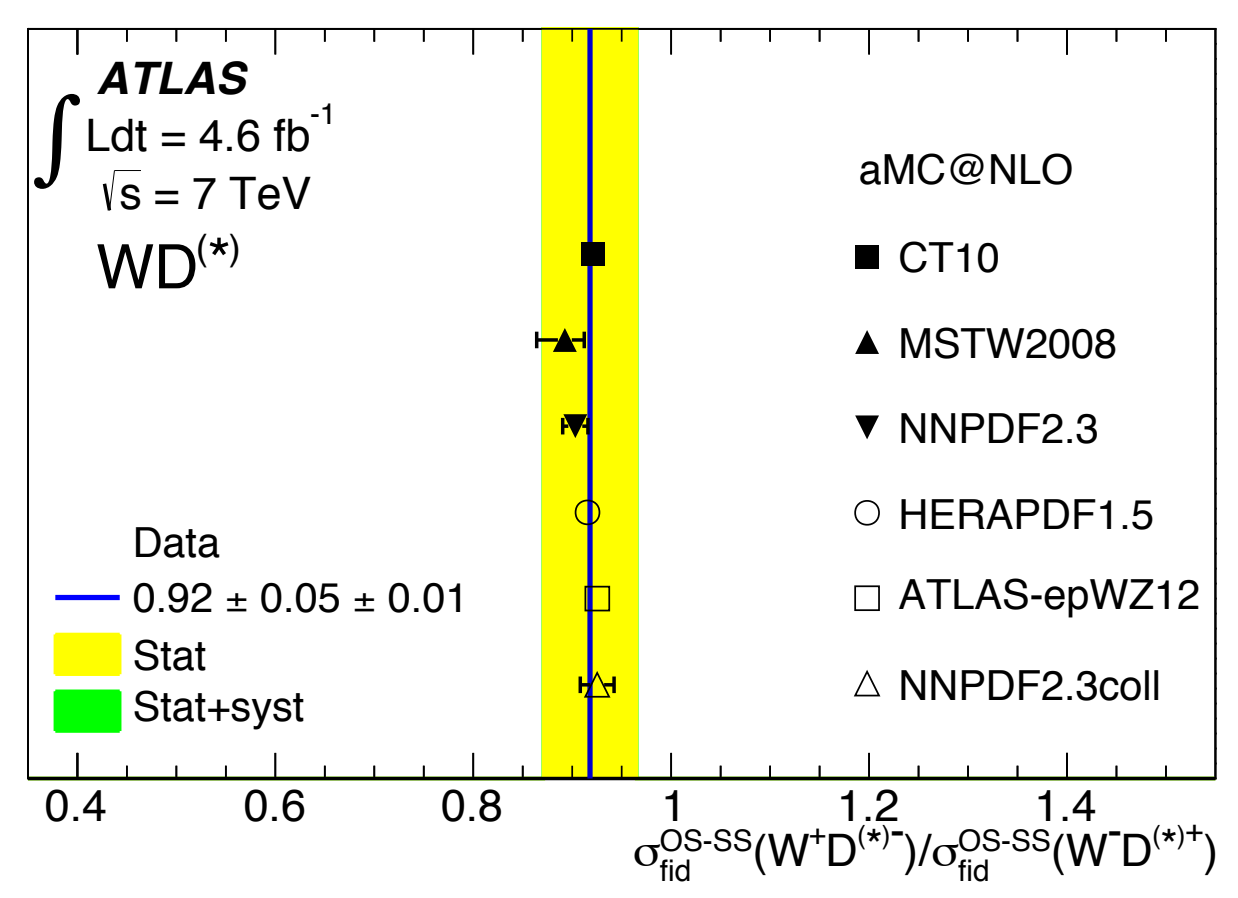

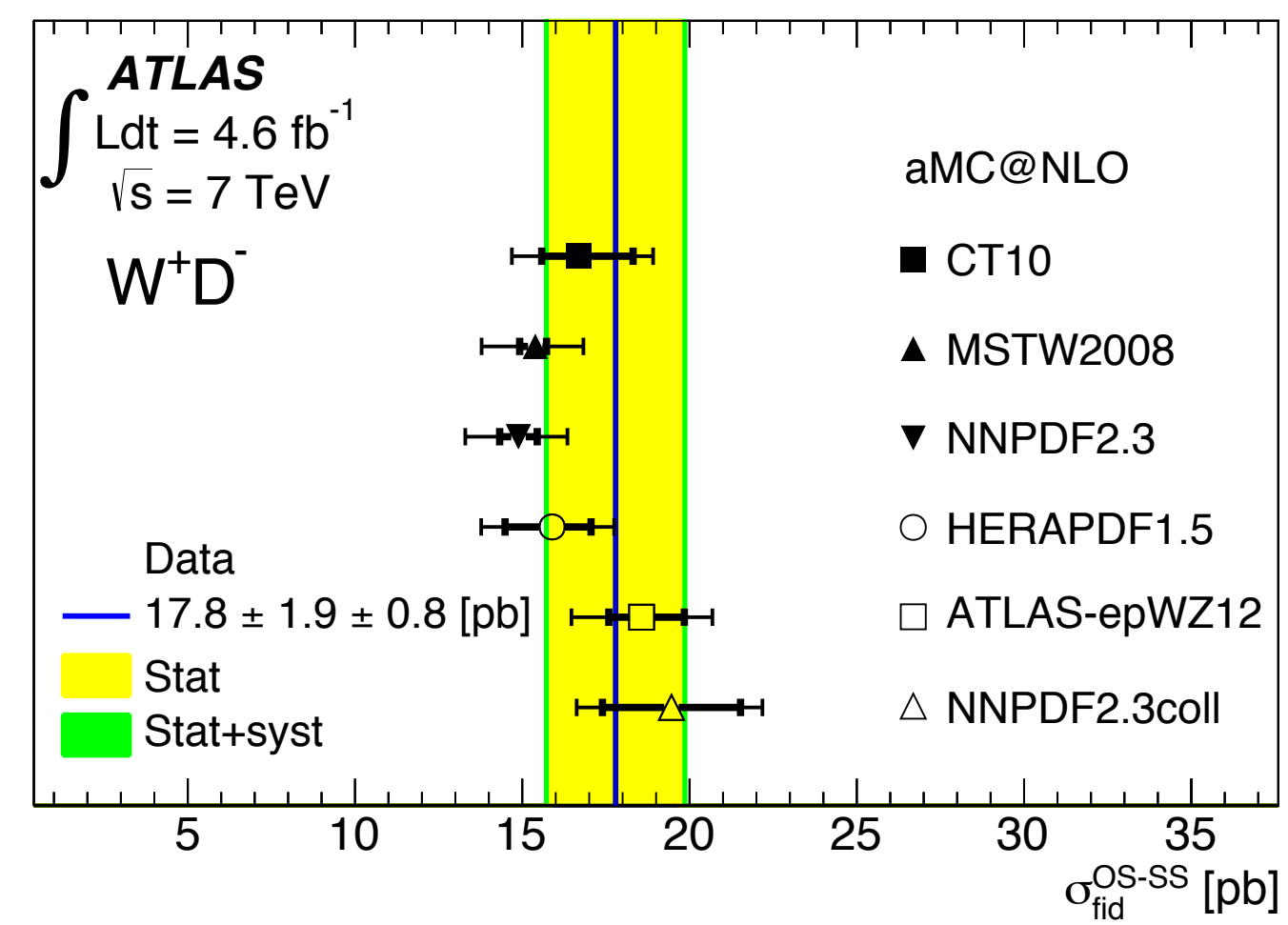




\section{W+charm: differential cross sections and strange quark PDF}
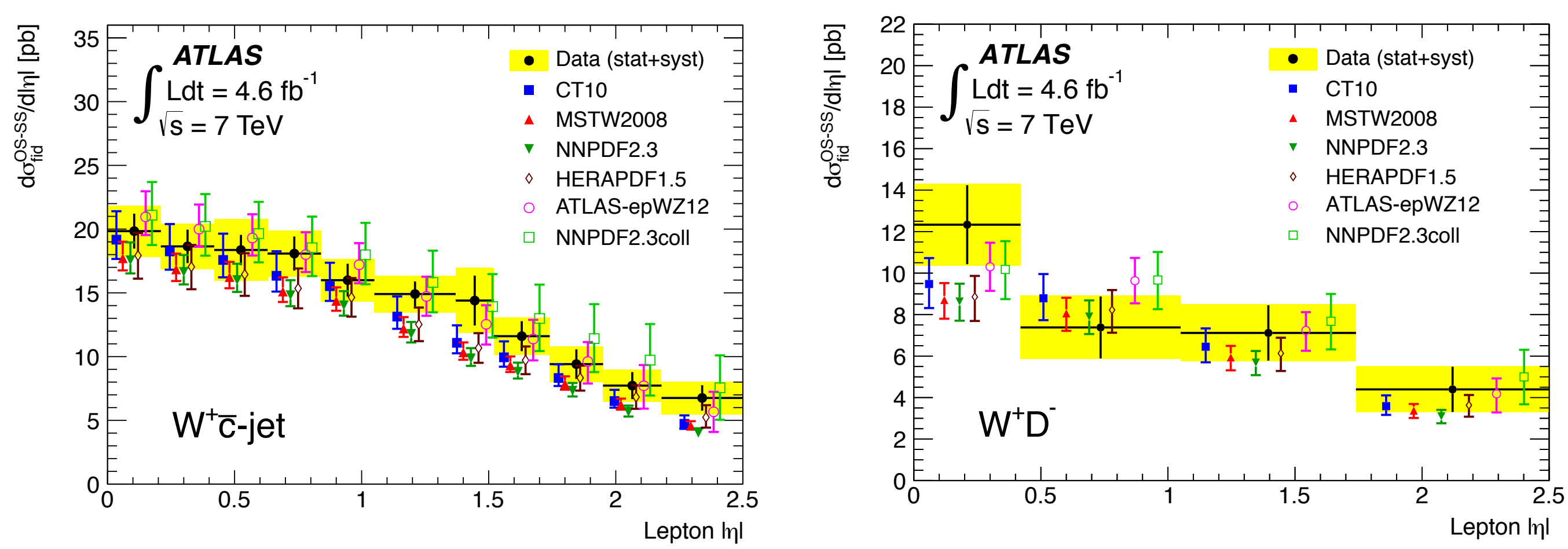

․ Measured also the lepton $|\eta|$ spectrum

口 Total fiducial cross sections used to test the strange quark content of the proton

■ Results consistent with unsuppressed strangeness scenario

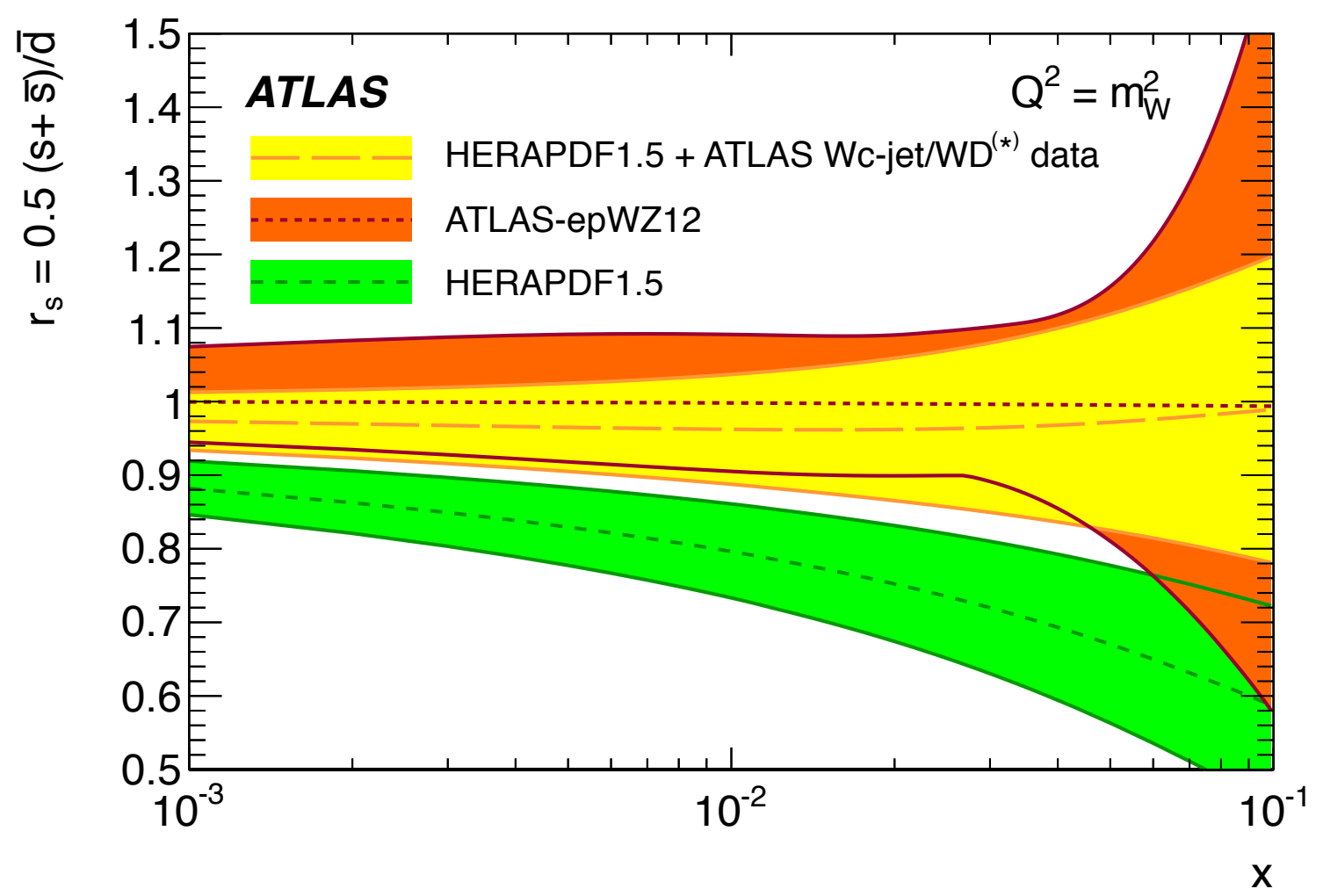




\section{Wb}

- Excess in the one jet bin more evident at high transverse momentum

- Experimental precision increase with a suitable signal definition (including the single top in the $\mathrm{W}+\mathrm{b}$ cross section)
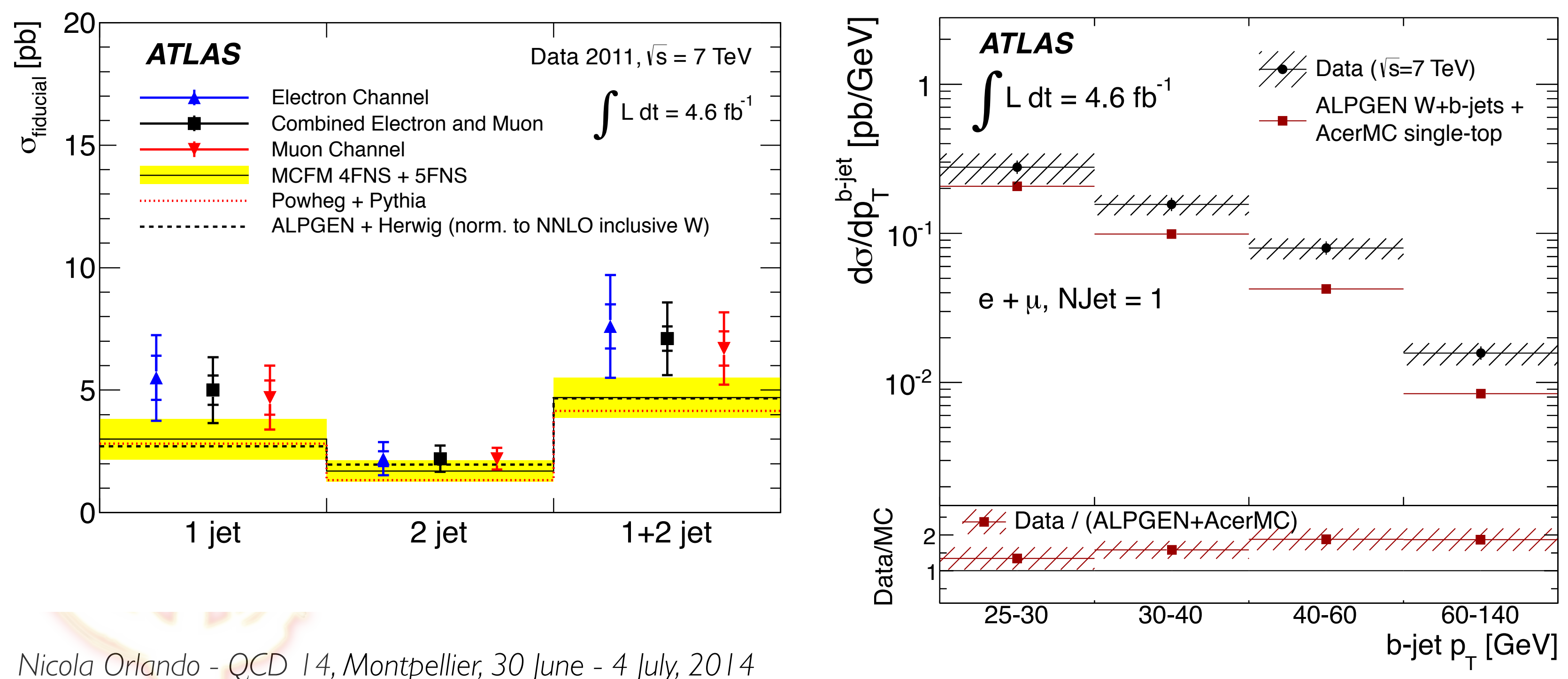

Nicola Orlando - QCD 14, Montpellier, 30 June - 4 July, 2014 


\section{$\mathbf{Z}+\mathbf{b}$ and $\mathbf{Z}+\mathbf{b b}$ : total fiducial cross sections}

Testing various implementations of QCD predictions

凹 Flavor number scheme (b-quark vs gluon initial state partons), partonshower, multileg LO vs NLO, b-quark mass sensitivity, ...

口 Z+b data favor NLO implementation in the 5FN scheme, Z+bb data favor 4FNS NLO

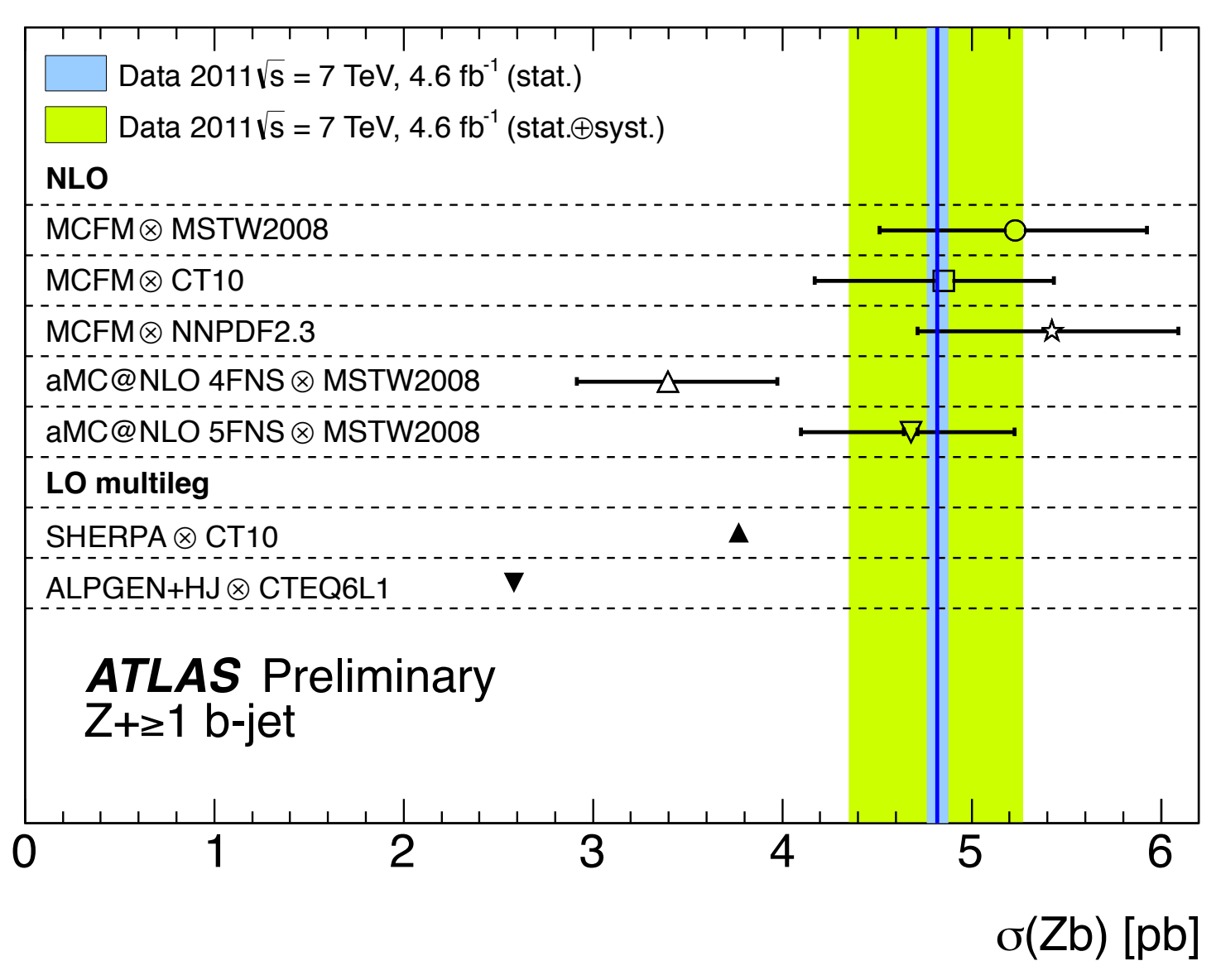

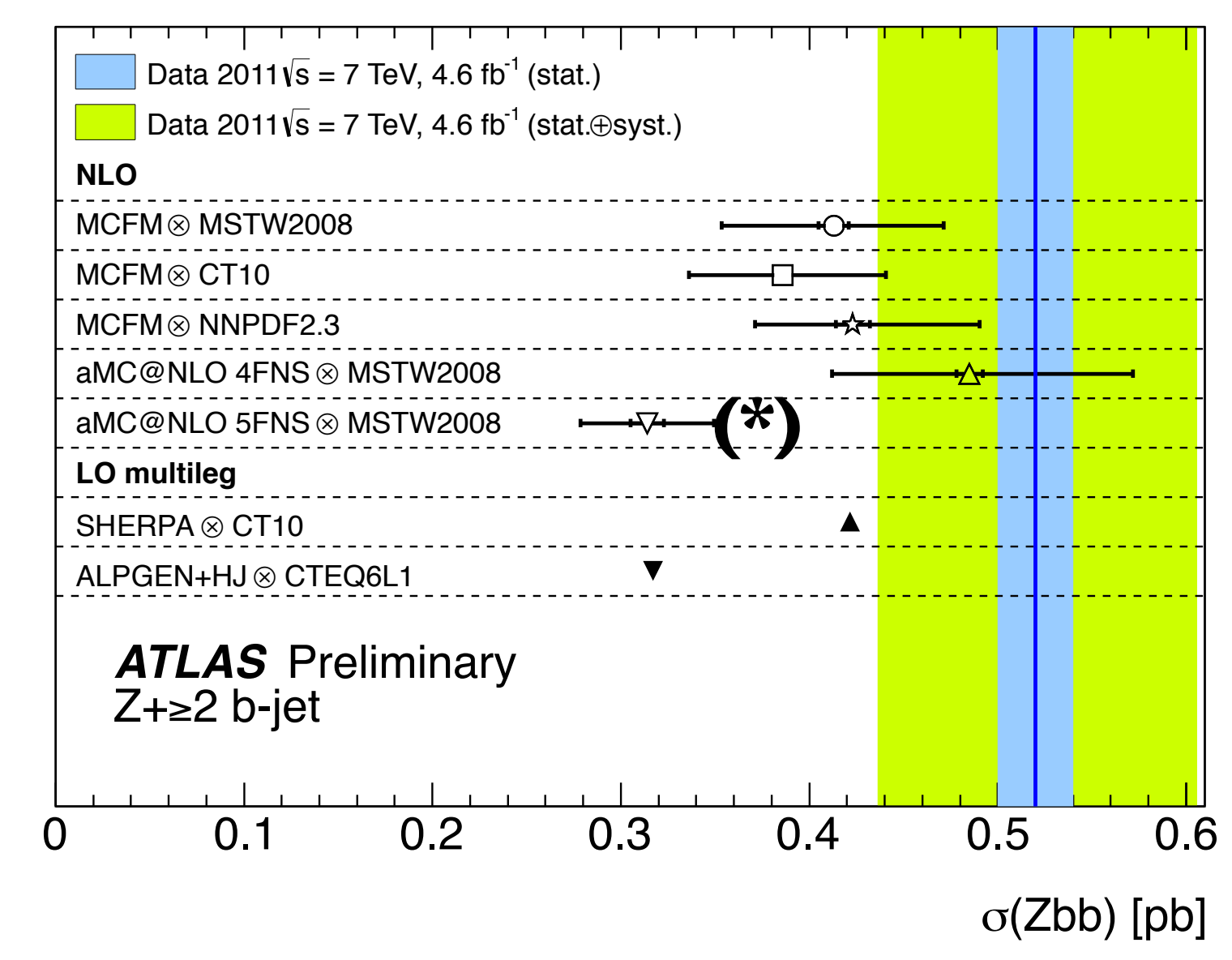

(*) Leading order for Z+bb final state 


\section{Z+b and Z+bb: differential cross sections}

- Comprehensive study of $Z$ and $b$-jets kinematics as well as $Z$-b and b-jets angular correlations

口 Evidence for limitations of the current theory predictions
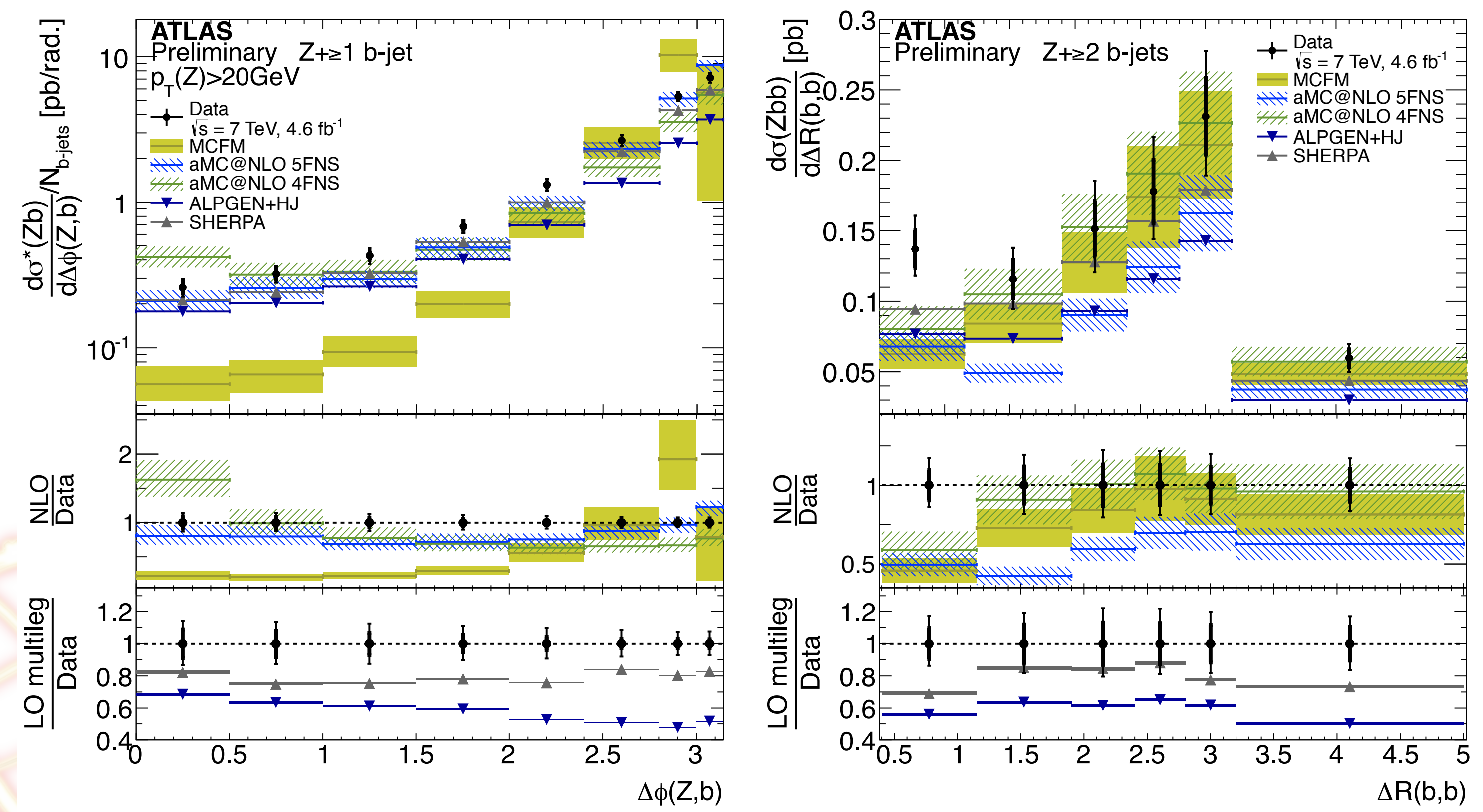


\section{Summary}

- Fruitful studies of inclusive vector bosons and vector boson plus jets recently published or under publication

$\square$ Improving experimental constraints on parton distribution functions

口 Increasing knowledge on Monte Carlo generators

- Systematic use of the data to validate/improve the theoretical predictions to be used in view of the physics reach of Run 2 at LHC 


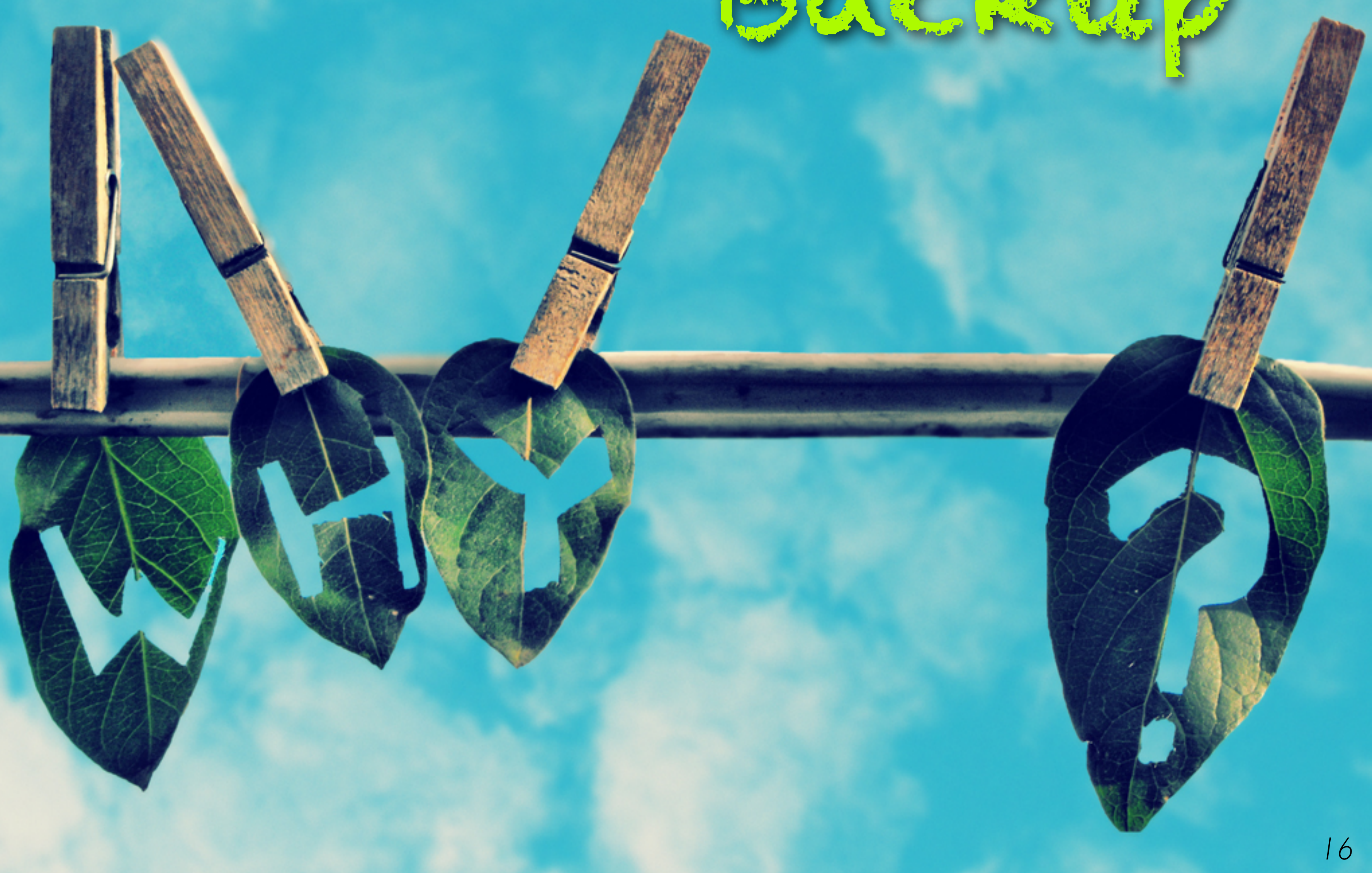




\section{$\mathbf{Z}$ boson transverse momentum}

口 Data compared also to several MC generators

口 Performed a tuning of parton shower parameters for PT $(Z) \lesssim 25 \mathrm{GeV}$

Data versus nominal $M C$ predictions

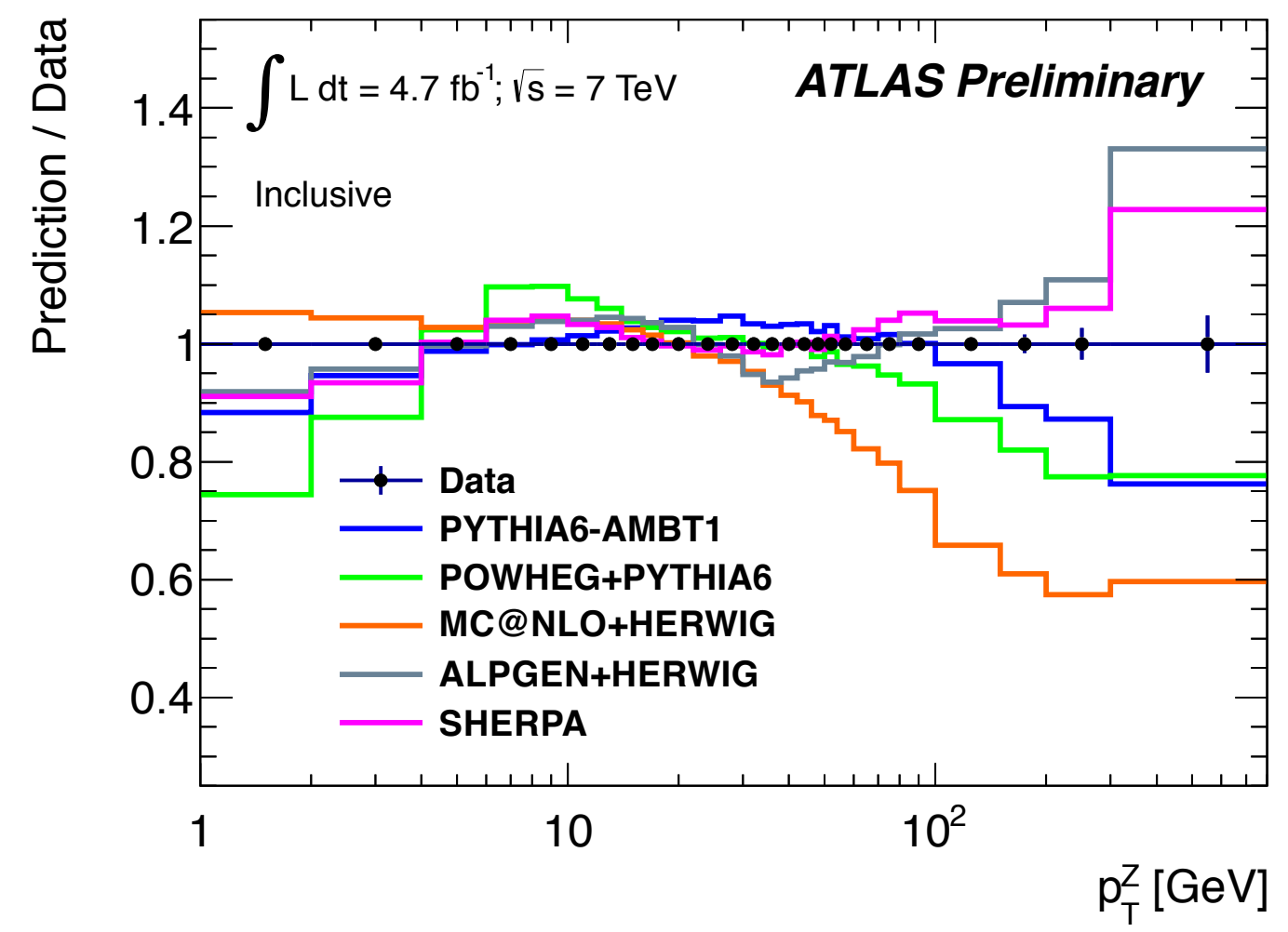

Comparison of the data with LO and NLO predictions using the base Pythia8 tune and the tune with the $\mathrm{PT}(Z)$ and $\Phi^{*}$ 20I I ATLAS data
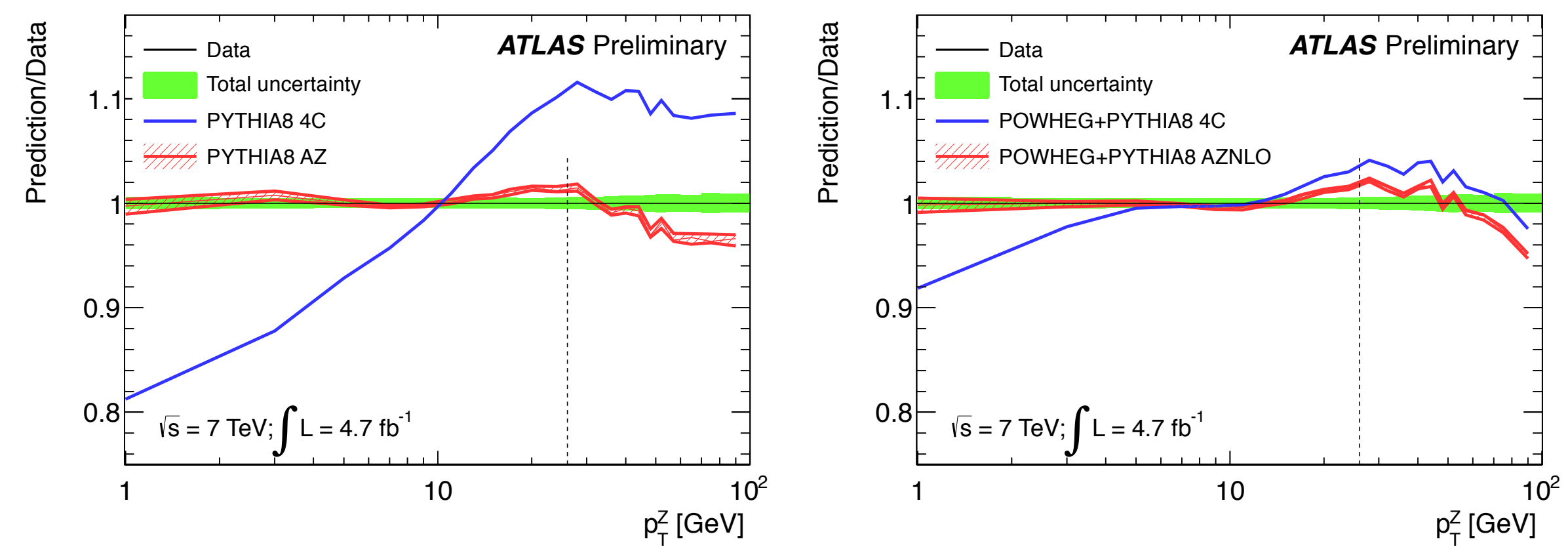


\section{Рт(Z): extra information}
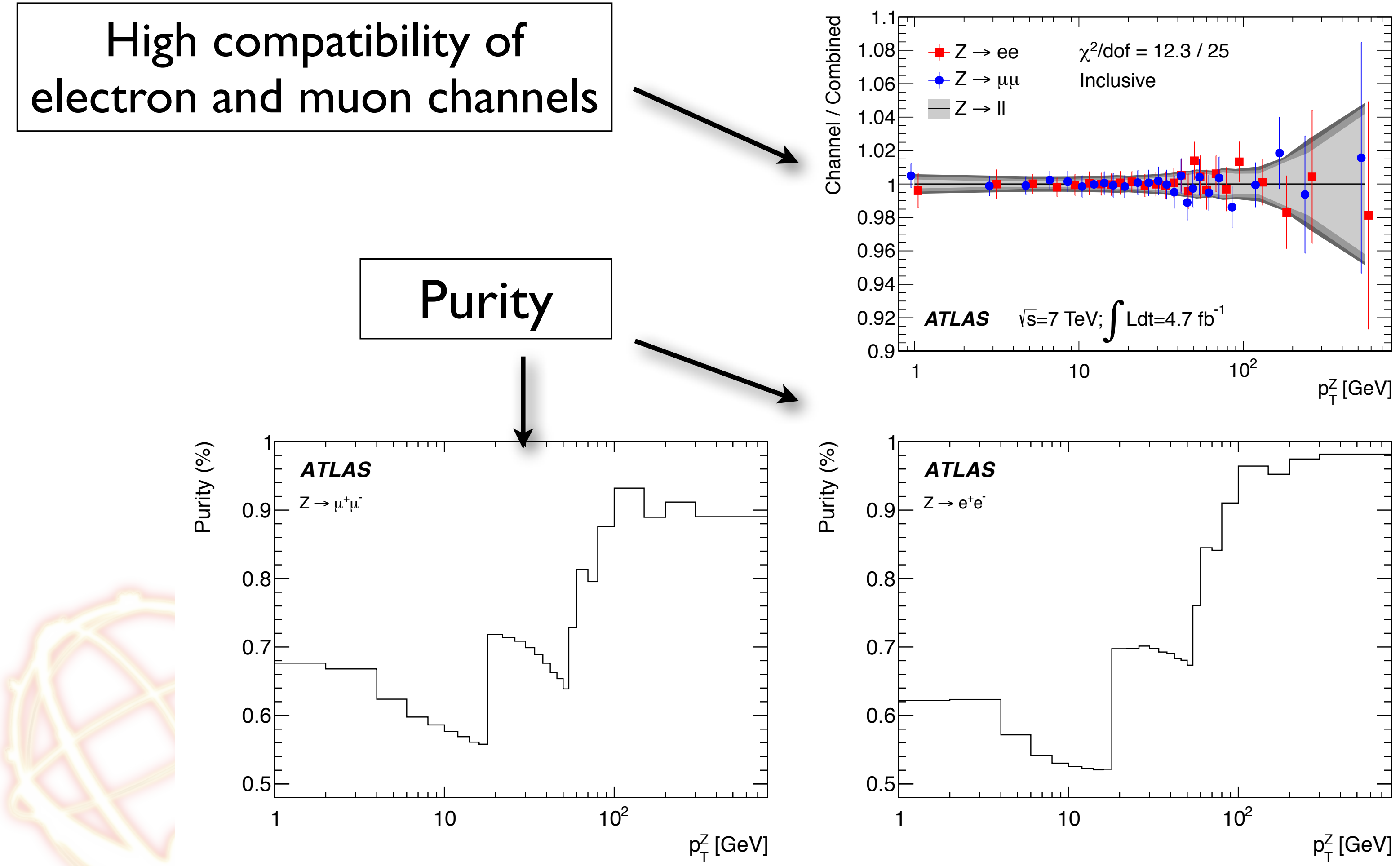

Nicola Orlando - QCD 14, Montpellier, 30 June - 4 July, 2014 


\section{Low mass neutral current Drell-Yan}

Before the QCD fit

\begin{tabular}{l|r|r}
\hline \hline Prediction & $\chi^{2}(8$ points $)$ & $\chi^{2}(6$ points $)$ \\
Nominal & Extended \\
\hline PoWHEG NLO+LLPS & $22.4(19.8)$ & $22.3(18.6)$ \\
FEWZ NLO & $48.7(28.6)$ & $139.7(133.7)$ \\
FEWZ NNLO & $13.9(12.9)$ & $7.1(7.1)$ \\
\hline \hline
\end{tabular}

口 Performed a QCD fit to the nominal and extended invariant mass spectra

口 Comparison data-NNLO improves, especially in the nominal analysis mass range
After the QCD fit

\begin{tabular}{l|r|r}
\hline \hline Prediction & $\chi^{2}$ (8 points) & $\chi^{2}$ (6 points) \\
& Nominal & Extended \\
\hline NLO Fit & 40.7 & 117.1 \\
NNLO Fit & 8.5 & 7.8 \\
\hline \hline
\end{tabular}

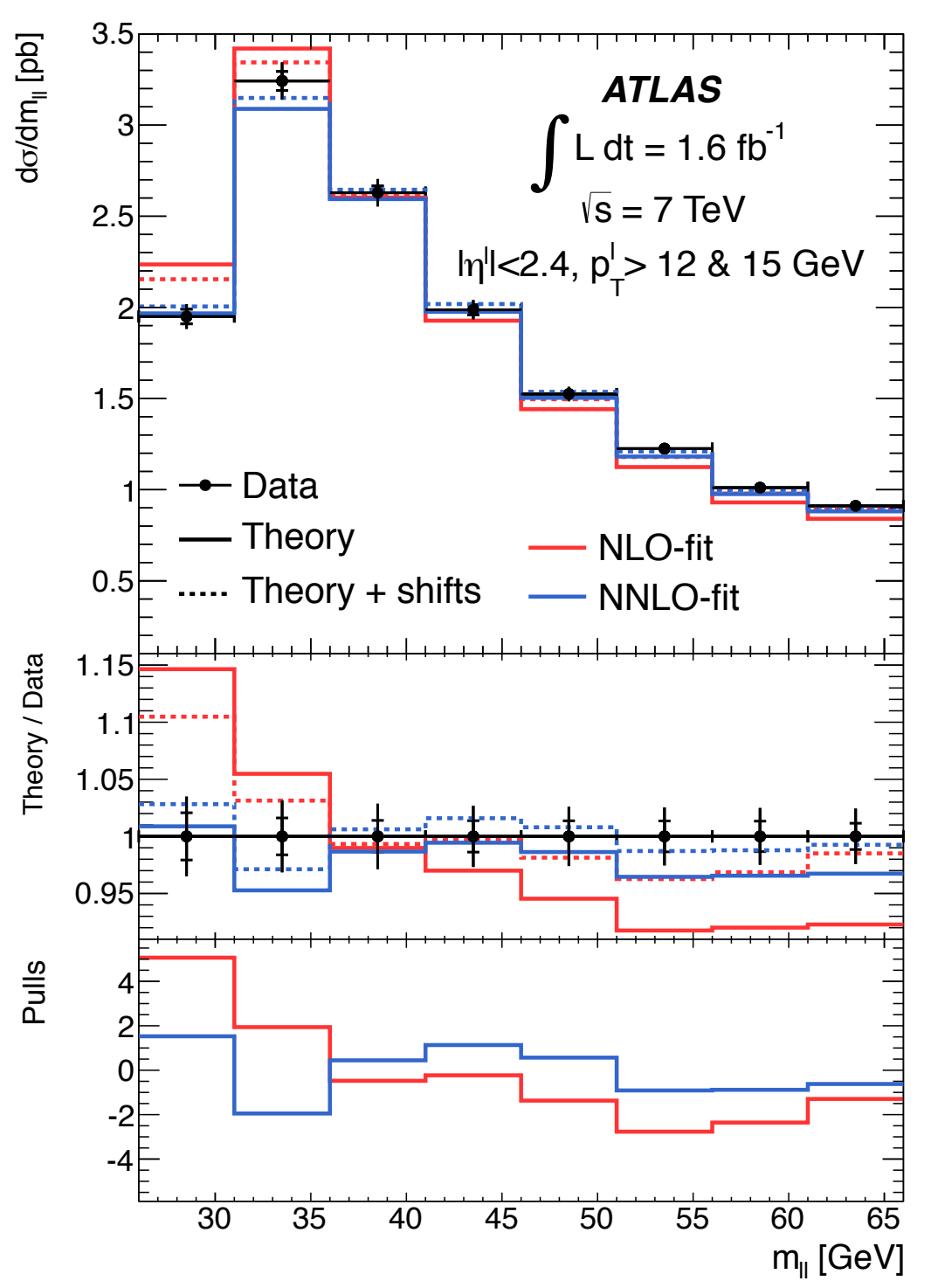

